\title{
Positive Breakdown Streamers and Acceleration in a Small Point-Plane Liquid Gap and Their Variation with Liquid Properties.
}

\author{
D. Linhjell, S. Ingebrigrtsen, L. E. Lundgaard \\ Sintef Energy Research, \\ NO-7465 Trondheim, Norway
}

\author{
Mikael Unge \\ ABB Corporate Research \\ SE-72178 Västerås, Sweden
}

\begin{abstract}
Positive breakdown streamers have been investigated in a short $(5 \mathrm{~mm})$ point-plane gap in cyclohexane and n-tridecane with and without the additives $\mathrm{N}, \mathrm{N}$-dimethylamine (DMA) with low ionization potential and azobenzene with a not quite as low ionization potential but a very low lowest excitation level. The effects off additives are fairly similar to what is known from longer gaps with DMA or pyrene added: the additives caused reduced breakdown voltage, significantly increased acceleration voltage and increased streamer velocity around breakdown. Molecule for molecule, azobenzene has less effect than DMA. The ratio between the acceleration and the breakdown voltage is significantly higher than in long gaps, though. Nonetheless, tests in short gaps are sufficiently good for screening (a large number of) liquid combinations.
\end{abstract}

The two base liquids without additives, and also neat trichloroethene and DMA have been tested with and without a tube causing spatial restriction of the streamer. Restriction causes increased breakdown voltage and promotes the fastest streamer modes.

\section{Introduction}

Streamers in oil are studied because of the relevance for decay and breakdown in oil-filled insulation systems, of which transformers represent one type. Point to plane gaps are usually used, mainly because it is possible to induce streamers with moderate voltages, but also because a more homogeneous gap will suppress many streamer phenomena which may take place if there are protrusions or more or less sharp particles present in an insulation system.

Small gaps, typically $5-10 \mathrm{~mm}$ but sometimes up to about $25 \mathrm{~mm}$ have been much used in such studies $[1,2,3]$. It is usually non-breakdown streamers that are studied in small gaps, and nonbreakdown streamers are usually of the slow type $(20 \mathrm{~m} / \mathrm{s}$ up to a few hundred meters per second) often designated $1^{\text {st }}$ mode streamers or a somewhat faster type $(1-3 \mathrm{~km} / \mathrm{s})$ designated $2^{\text {nd }}$ mode [4]. In large gaps (typically $50 \mathrm{~mm}$ or more) above breakdown voltage $\left(\mathrm{V}_{\mathrm{b}}\right)$ one has observed faster streamers of $3^{\text {rd }}$ mode (ill-defined in some liquids, but usually in the range $4-20 \mathrm{~km} / \mathrm{s}$ ) and $4^{\text {th }}$ mode (usually significantly faster, often $70-200 \mathrm{~km} / \mathrm{s}$ for positive streamers) bridging the final part of or the whole gap in a "fast event" $[4,5,6,7,8]$.

In a given geometry, transition voltages between modes are liquid dependent. For positive streamers (from a positive point electrode) in typical mineral transformer oils the transition to the fast modes takes place at what is referred to as "acceleration voltage", $\mathrm{V}_{\mathrm{a}}$ which is typically about twice the breakdown voltage from a sharp point anode[4,8]. In many other liquids, some of which are considered as more environmentally friendly replacements for mineral oil, the transition takes place only few per cent above breakdown voltage [9]. This has consequences for a practical insulation system's response to overvoltages of limited duration: a fairly slow streamer may not bridge the available gap in the duration of the overvoltage, while a $4^{\text {th }}$ mode streamer often will. Thus the study of which aspects of the liquid influences the difference between $V_{a}$ and $V_{b}$ has become of practical importance, and this has usually been done in long gaps, also in our laboratories $[5,6,7,8]$. It has been observed that a high degree of branching of $2^{\text {nd }}$ mode streamers increases $V_{a}[1,5,6,7]$, and so far it seems that a small concentration of constituents or additives with significantly lower ionisation potential than the bulk of the liquid leads to increased branching $[1,3,5,6,7]$.The liquid dependent branching tendency of typically nonbreakdown streamers can be studied in small gaps $[1,3]$. Since one wants to vary electronic properties of the liquid these studies often involve additives that are expensive, toxic or both and which are therefore more conveniently handled in a small test cell.

This paper presents a study of breakdown streamers where the transition to fast streamers $\left(3^{\text {rd }}\right.$ and $4^{\text {th }}$ modes) for the first time is studied in a gap as small as $5 \mathrm{~mm}$. Different base liquids have been tested with varying concentration of additives, and two liquids normally used as additives have also been tested as base liquids. For some liquids the effect of spatially restricting the streamers with a tube (limiting how much branching is possible) has been studied. 


\section{Experimental}

The PTFE test cell is shown in Figure 1 and the electrode arrangement in Figure 2. The oil volume is $30 \mathrm{ml}$, the gap length $5 \pm 0.3 \mathrm{~mm}$, and the point is electrochemically etched to a somewhat hyperbolically shape with a minimum radius of curvature of about $10 \mu \mathrm{m}$ at the start of a test sequence. An experimental sequence with one liquid wears the point down to $50 \mu \mathrm{m}$, so it is replaced each time the test cell is filled. The wear has no consequences since this is a breakdown and acceleration experiment as long as initiation takes place at a voltage well below breakdown. The plane diameter is $13 \mathrm{~mm}$.

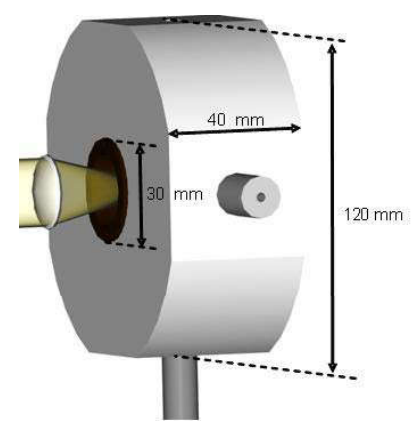

Figure 1. Liquid test cell mad from PTFE.
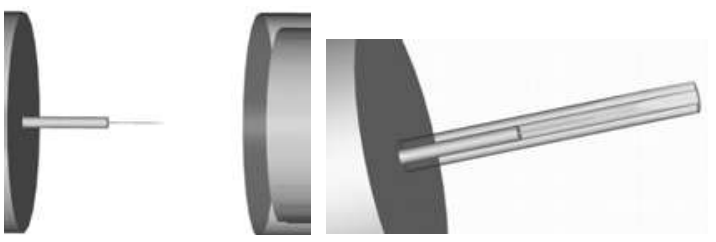

Figure 2. Electrode arrangement (left) and restricting tube arrangement (right).

For the experiments with spatial restriction of the streamers, a PTFE tube with inner diameter 0.76 $\mathrm{mm}$ and outer diameter $1.6 \mathrm{~mm}$ was used (Figure 2 ). The single stage impulse generator generates a square impulse with a somewhat voltage-dependent $10-90 \%$ rise time (about $25 \mathrm{~ns}$ at $30 \mathrm{kV}$, increasing to $35-45 \mathrm{~ns}$ at $75 \mathrm{kV}$ ) and having a duration of 50 $\mu \mathrm{s}$.

The test sequence is automated except for some manual work with removing gas bubbles produced in the tube in the experiments with spatial restriction, and runs from a minimum to a maximum set voltage in set steps (usually $5 \mathrm{kV}$ ) with 3 minutes interval between each impulse and usually 15 impulses per voltage level. The only measurement is the voltage waveform by means of a Tektronix TDS 540 oscilloscope. The record is automatically stored, and the following automated measurements are done: step amplitude, breakdown yes/no and time to breakdown. From time to breakdown, an average streamer velocity across the gap is calculated. This is a minimum streamer velocity since there may be a delay from the streamer reaches the plane till the voltage actually collapses. On the other hand, streamer start is assumed to be just where the steep impulse front starts to flatten, which may be a little late at the higher voltages, possibly leading to streamer velocities somewhat on the high side. This approach means that velocity is calculated for breakdown streamers only.

One complete test sequence is run in the same liquid without changing or filtering. Thus there is a build-up of carbon particles in the liquid as the test sequence progresses. One long gap study indicates that this has no consequences for positive streamers [10], while another long-gap study shows significant changes in both acceleration voltage and breakdown voltage [6], so there is a possibility that the results may be influenced by the particles.

Table 1 shows a list of liquid combinations used and Table 2 lists the purity and some relevant electronic properties of the liquids, calculated with quantum chemical methods $[11,12]$. The table shows zero-field gas phase values, which may not be the relevant values, since the ionization potential is typically lowered about $1 \mathrm{eV}$ in the liquid state [13] and is highly dependent upon the local electric field $[11,12,14]$. The excitation levels are in most cases much less dependent upon the field. Where more than one value is listed, it is because $\mathrm{IP}_{1}$ also depends upon the orientation of the molecule relative to the field.

Table 1. Liquid combinations and spatial restrictions (tube).

\begin{tabular}{|c|c|c|c|c|c|}
\hline $\begin{array}{l}\text { Base } \\
\text { liquid }\end{array}$ & & & & & $\begin{array}{l}\text { Also } \\
\text { with } \\
\text { tube }\end{array}$ \\
\hline $\begin{array}{l}\text { Cyclo- } \\
\text { hexane }\end{array}$ & & & & & $\bar{X}$ \\
\hline $\begin{array}{l}\text { n-Tri- } \\
\text { decane }\end{array}$ & & & & & $\bar{X}$ \\
\hline $\begin{array}{l}\text { Trichloro- } \\
\text { ethene }\end{array}$ & & & & & $\bar{X}$ \\
\hline DMA & None & 0 & 0 & 0 & $\mathrm{X}$ \\
\hline
\end{tabular}

$\mathrm{DMA}=\mathrm{N}, \mathrm{N}$-dimethylaniline, $\mathrm{AZO}=$ azobenzene 
Table 2. Purity and selected gas-phase electronic properties of base liquids and additives.

\begin{tabular}{|l|cccc|}
\hline $\begin{array}{l}\text { Base liquid } \\
\text { or } \\
\text { additive }\end{array}$ & $\begin{array}{c}\text { Purity } \\
\text { at } \\
\text { least }\end{array}$ & $\begin{array}{c}\text { Ioni- } \\
\text { zation } \\
\text { potent- } \\
\text { ial } \\
\text { (IP) }\end{array}$ & $\begin{array}{c}\text { Lowest } \\
\text { elec- } \\
\text { tronic } \\
\text { excit- } \\
\text { ation } \\
\text { level } \\
(\mathrm{EX})\end{array}$ & Ref. \\
& & & & \\
{$[\mathrm{eV}]$} & \\
\hline \hline Cyclohexane & 99.9 & 9.7 & 6.4 & {$[11]$} \\
n-Tridecane & 99 & 9.0 & 6.28 & {$[11]$} \\
$\begin{array}{l}\text { N,N- } \\
\text { dimethylaniline }\end{array}$ & 99 & 7.42 & 4.03 & {$[12]$} \\
$\begin{array}{l}\text { Trichloroethene } \\
\text { Azobenzene }\end{array}$ & 99.5 & 9.17 & 4.97 & {$[12]$} \\
& 99 & $7.8-8.6$ & 2.21 & {$[12,15-$} \\
& & & & $18]$ \\
\hline
\end{tabular}

$\mathrm{N}, \mathrm{N}$-dimethylaniline (DMA) was selected as an additive because of its low ionization potential relative to the base liquids. Azobenzene (AZO) was selected to investigate if the low lowest excitation level could have an effect, although it too has an ionization potential that is lower than for cyclohexane and n-tridecane, but not as low as DMA. Trichloroethene is included because it shows a significant effect on negative streamers in long gaps, presumably because of its effect as an electron scavenger $[3,5,6]$, and usually little effect on positive streamers although some reduction in acceleration voltage has been observed $[6,7]$.

\section{Results}

Despite being a small gap, breakdown and acceleration is observed. For some tested liquid/additive combinations where the additive has an effect upon acceleration, the higher concentrations tested pushed the acceleration voltage beyond the voltage capability of the impulse generator.

An example of a breakdown streamer velocity diagram is shown in Figure 3. At the lower voltages there are few points because not all impulses led to breakdown. Without spatial restriction, some streamers are becoming fast at about $56 \mathrm{kV}$. For all liquids tested, spatial restriction increases the breakdown voltage and reduces the acceleration voltage as shown in Figure 4. For trichloroethene and dimethylaniline without spatial restriction, no clear acceleration voltage was observed, indicating that it might be above the voltage range of the setup. Apparently, the acceleration voltage with spatial restriction is much lower than the breakdown voltage. This is an artefact of definitions, as the breakdown voltage given is the $50 \%$ value while the acceleration voltage is the velocity plot knee point resulting from just a few streamers being fast, and with spatial restriction fast streamers occur already from breakdown, even below the 50\% breakdown voltage.

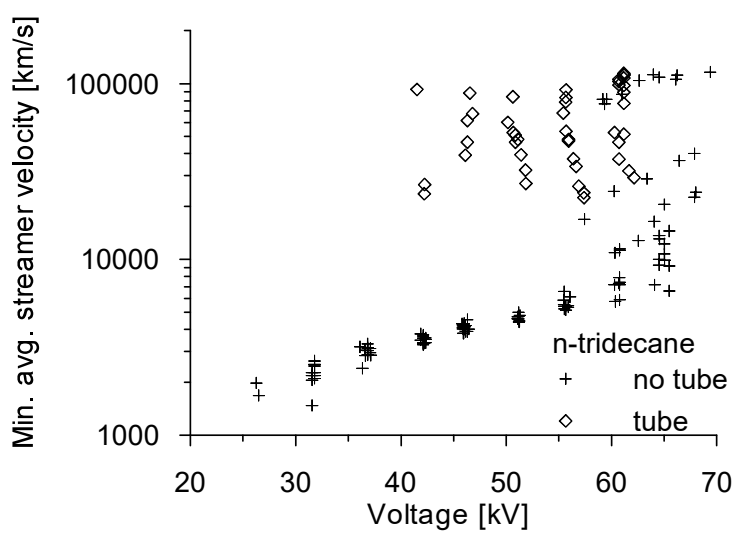

Figure 3. Example of streamer velocity plot for two experimental sequences, n-tridecane with and without tube (i.e. spatial restriction).

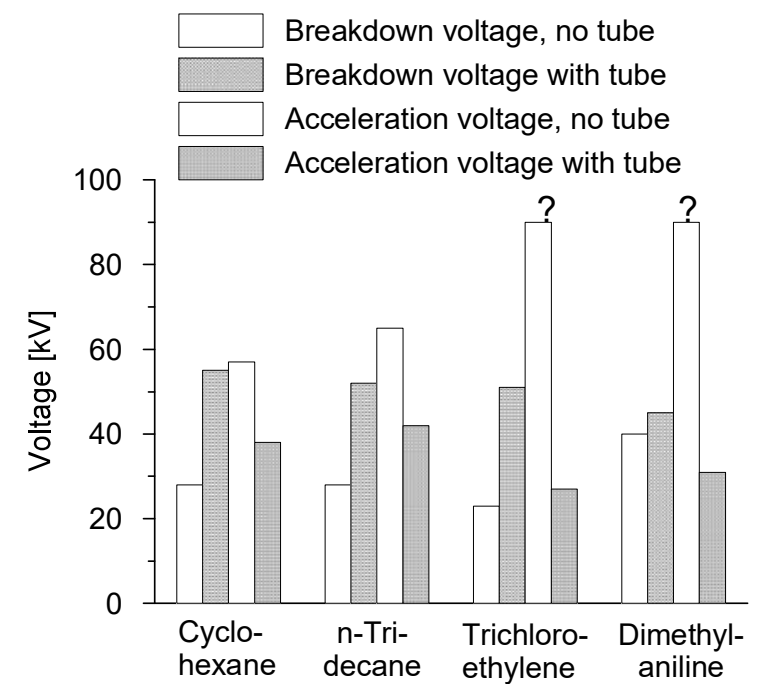

Figure 4. Breakdown and acceleration voltage with and without spatial restriction.

Although Figure 3 gives the impression that all spatially restricted breakdown streamers are very fast, this is not universal. In cyclohexane and TCE restricted streamers slower than $10 \mathrm{~km} / \mathrm{s}$ are very rare (just 2 registered in each liquid) while in DMA restricted streamer velocities occupy the full range from about $700 \mathrm{~m} / \mathrm{s}$ up to about $100 \mathrm{~km} / \mathrm{s}$ down to the lowest voltages causing breakdown.

A typical velocity plot with additives is shown in Figure 5. The type of effect is the same in both cyclohexane and n-tridecane with both the low ionization potential additive DMA and the low excitation level additive azobenzene: somewhat increased streamer velocity at low voltages around and slightly above breakdown (Figure 6), at least at the higher additive concentrations, higher acceleration voltage (Figure 7) and, since the streamer velocity continues to increase slowly up to the acceleration voltage, the velocity at acceleration 
generally increases, too (Figure 8). One can also see from the figures that for the same increase in one of these parameters, it requires fewer molecules of DMA than azobenzene added.

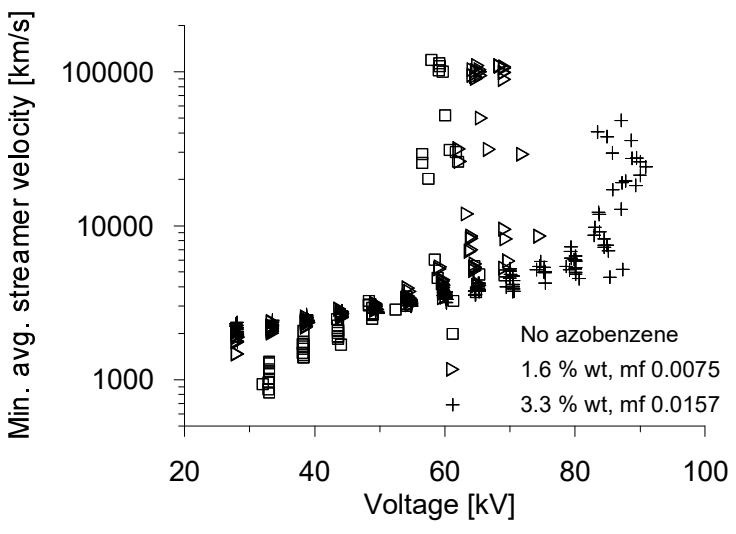

Figure 5. Streamer velocities in cyclohexane with azobenzene added. $\mathrm{mf}=$ mole fraction.

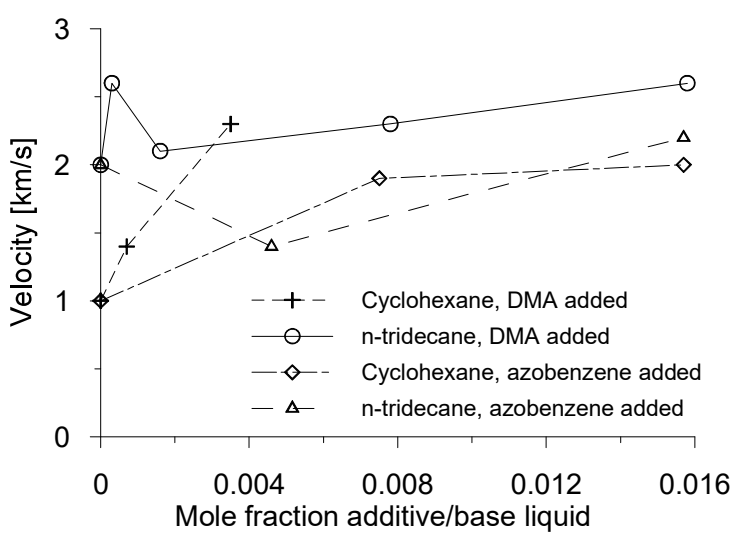

Figure 6. Average streamer velocity at the lowest breakdown voltages.

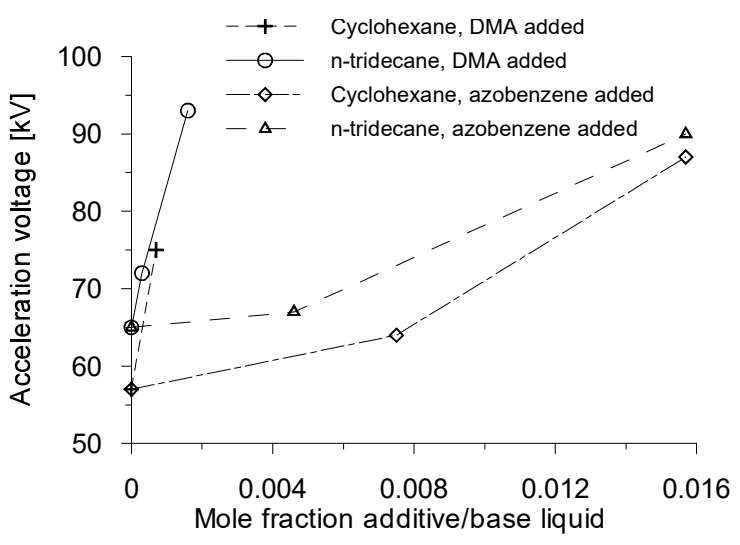

Figure 7. Acceleration voltage vs. additive mole fraction.

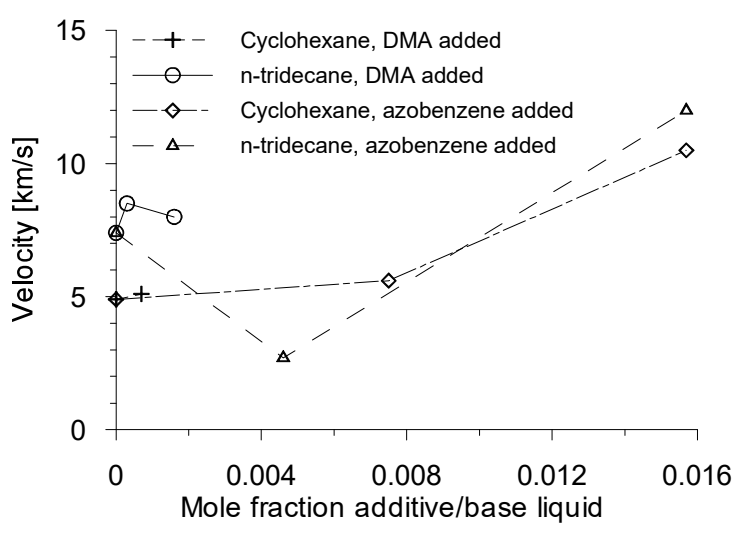

Figure 8. Streamer velocity at acceleration.

Figure 9 shows that the trend for the breakdown voltage is less clear, although at high enough concentration of additive there is a clear downward trend.

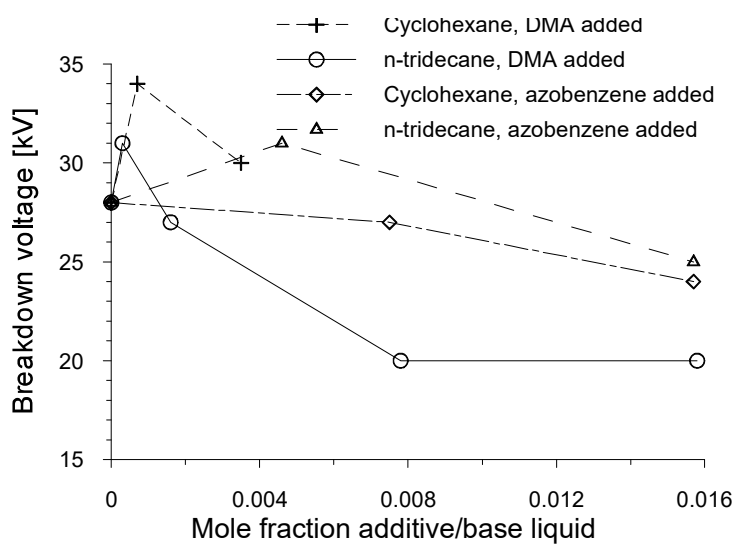

Figure 9. Breakdown voltage vs. additive mole fraction.

\section{Discussion}

The behaviour of the breakdown and acceleration voltages with increasing amount of low ionization potential or low excitation potential additives in cyclohexane and n-tridecane is in line with what has been observed in long $(50-80 \mathrm{~mm})$ gaps, with pyrene added to cyclohexane [1] or DMA added to cyclohexane or to various low-aromatic mineral oils $[5,6,7]$. With increasing acceleration voltage and generally decreasing breakdown voltage, the ratio between them also increases as shown in Figure 10. The ratios observed in the short gap are much higher than in the long gaps, though: in a long gap, cyclohexane without additive has a ratio about 1.1, increasing to 2.5 with $1 \%(0.064 \mathrm{M}$, mole fraction $0.007)$ DMA, or to 2.1 with $0.1 \mathrm{M}$ pyrene). The reason probably is that $2^{\text {nd }}$ mode streamers seem to be considerably more branched in short gaps [3] than in long gaps [5], and extensive branching seems to suppress the transition to faster modes, 
presumably because of the mutual shielding keeping the tip fields comparatively low.

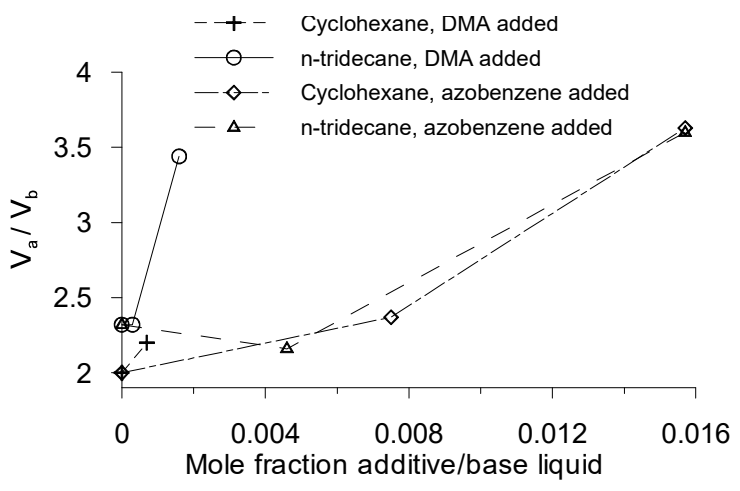

Figure 10. Ratio between acceleration and breakdown voltage.

According to Figure 8, the streamer velocities just before acceleration are so high that one can question what modes are present. Usually $2^{\text {nd }}$ mode is not considered to be quite as fast as what the figure shows. In long gaps, the velocity just before acceleration is $4.5 \mathrm{~km} / \mathrm{s}$ or lower, even with $1 \%$ (0.064M)DMA added [5,6,7]. Most likely part of the gap is crossed as $3^{\text {rd }}$ mode. The uncertainty cannot be resolved here due to the lack of photographic evidence.

As expected, spatially restricting the streamers with a tube led to considerably increased velocities. This is consistent with spatial restriction largely removing the extensive branching and mutual shielding and thus promoting faster modes.

Trichloroethene has a high acceleration voltage (Figure 4). This came as a surprise, because its effect upon positive streamers in long gaps when used as an additive is to reduce acceleration voltage [6,7]. Similarly, DMA used alone had a high breakdown voltage, but used as an additive in long gaps it often but not always causes a reduction in breakdown voltage.

Azobenzene was included as an additive because of a hypothesis that the lowest electronic excitation level might have an influence $[11,12,19]$, particularly at the higher local fields which may exist in front of a streamer tip, since the ionisation potential may be so reduced with increased field $[10,11]$ that it approaches the lowest excitation level. However, in this experiment it has an effect similar to but weaker than with DMA, and this experiment alone cannot tell if the effect is caused by the low excitation level or the ionization potential which is lower than in the base liquid but still higher than in DMA. That the effect is weaker than in DMA, which has a lowest excitation level almost twice as high as azobenzene, might indicate that it is not the excitation level which is important, but this is contrary to the discussion in a paper about a long gap experiment with a natural ester as base liquid [19].

\section{Conclusion}

Breakdown and acceleration voltages in short electrode gaps show the same development with increasing concentration of low ionization potential additives (or possibly low excitation potential additives) as in long gaps, although the ratio between acceleration and breakdown voltages is very much higher than in the long gaps. The additives N,N-dimethylaniline and azobenzene cause slightly reduced breakdown voltage, significantly increased acceleration voltage, and increased streamer speeds at the lower voltages leading to breakdown. The streamer development in the small and the large scale experiments is sufficiently similar so that small scale tests can be used to screen potential additive candidates before eventually testing the more successful ones in a long gap.

This is an activity in progress, and more combinations of base liquids and additives will hopefully give more answers.

\section{Acknowledgements}

This work has been supported by The Norwegian Research Council, ABB and Statnett.

\section{References}

1. O. Lesaint and M. Jung, "On the relationship between streamer branching and propagation in liquids: influence of pyrene in cyclohexane", J. Phys. D: Appl. Phys,, Vol. 33 (2000), pp. 1360-1368.

2. J. C. Devins, S. J. Rzad and R. Schwabe, "Breakdown and prebreakdown phenomena in liquids", J. Appl. Phys, Vol. 52, No. 7, July 1981, pp. 4531-4545.

3. S. Ingebrigtsen, H. S. Smalø, P.-O. Åstrand and L. E. Lundgaard, "Effects of Electron-attaching and Electron-Releasing Additives on Streamers in liquid Cyclohexane", IEEE Trans. Diel. and El. Ins., Vol. 16, No. 6, Dec. 2009, pp. 15241535.

4. O. Lesaint, "Propagation of positive discharges in long liquid gaps", Proc. IEEE 12th Int. Conf. on Cond. and Breakd. in Diel. Liquids (ICDL), Rome, Italy, July 15-19 1996, pp. 161166.

5. D. Linhjell, S. Ingebrigtsen, L. E. Lundgaard and M. Unge, "Streamers in Long Point-Plane Gaps in cyclohexane with and without Additives", Proc. IEEE 17th Int. Conf. on 
Cond. and Breakd. in Diel. Liquids (ICDL), Trondheim, Norway June 26-30 2011.

6. N. V. Dung, H. K. Høidalen, D. Linhjell, L. E. Lundgaard and $M$. Unge, "Influence of Impurities and Additives on Positive Streamers in Paraffinic Model Oil", IEEE Trans. Diel. and El. Ins., Vol. 19, No. 5, Oct. 2012, pp. 1593-1603.

7. N. V. Dung, H. K. Høidalen, D. Linhjell, L. E. Lundgaard and M. Unge, "Effects of reduced pressure and additives on streamers in white oil in long gap", Submitted (2012) for publ. in J. Appl. Phys.

8. L. Lundgaard, D. Linhjell and G. Berg, "Propagation of Positive and Negative Streamers in Oil with and without Pressboard Interfaces", IEEE Trans. Diel. and El. Ins., Vol. 5, No. 3, June 1998, pp. 388-395.

9. C. Tran Duy, O. Lesaint, A. Denat and N. Bonifaci, "Streamer Propagation and Breakdown in Natural Ester at High Voltage", IEEE trans. Diel and El. Ins., Vol. 16, No. 6, 2009, pp. 1582-1594.

10. O. Lesaint, A. Saker, P. Gournay, R. Tobazeon, J. Aubin and M. Mailhot, "Streamer Propagation and Breakdown under AC Voltage in Very Large Oil Gaps", IEEE Trans Diel. and El. Ins., Vol. 5, No. 3, 1998, pp. 351-359.

11. H. S. Smalø, Ø. Hestad, S. Ingebrigtsen and P.O. Åstrand, "Field dependence on the molecular ionization potential and excitation energies compared to conductivity models for insulation materials at high electrical fields", J. Appl. Phys 109, 073306 (2011).

12. N. Davari, P.-O. Åstrand, S. Ingebrigtsenand M. Unge, "Excitation energies and ionization potentials at high electric fields for molecules relevant for electrically insulating liquids", J.Appl. Phys. 113, 143707 (2013)

13. H. S. Smalø, P.-O. Åstrand and S. Ingebrigtsen, "Calculation of Ionization Potentials and Electron Affinities for Molecules Relevant for Stremaer Initiation and Propagation", IEEE Trans. Diel and El. Ins., Vol. 17,2010, pp. 733-741.

14. G. V. Marr, "Photoinization Processes in Gases", Academic Press, New York, 1967, Libr. Of Congress No. 66.-30092.

15. H. S. Smalø, P. O. Aastrand and S. Ingebrigtsen, "Calculation of Ionization Potentials and Electron Affinities for Molecules Relevant for Streamer Initiation and Propagation", IEEE Trans. Diel and Electr. Insul. Vol 17, No. 3, pp. 733 -741 (2010).

16. T. Schultz, J. Quenneville, B. Levine, A. Tonioli, T. J. Martinez, S. Lochbrunner, M. Schmitt, J. P. Shaffer, M. Z. Zgierski and A. Stolow, "Mechanism and Dynamics of Azobenzene Photoisomerization", J. Am. Chem Soc. Vol. 125, No. 27, 8098 (2003).
17. N. E. Petrachenko, V. I. Vovna and G. G. Furin, "Mechanism and Dynamics of Azobenzene Photoisomerization", J. of Fluorine Chemistry, Vol. 63 No. 1-2, 85 (1993).

18. M. L. Tiago and J. R. Chelikowsky, "Firstprinciples GW-BSE excitations in organic molecules", Solid State Communications Vol 136, No. 6, pp. 333-337 (2005).

19. M. Unge, S. Singha, N. V. Dung, D. Linhjell, S. Ingebrigtsen, L. E. Lundgaard: "Enhancements in the lightning impulse breakdown characteristics of natural ester dielectric liquids". Submitted for publication in Applied Physics Letters. 